\title{
REPRESENTACIÓN SOCIAL DEL RIESGO EN LA COSTA DE OAXACA: AGROTÓXICOS, SALUD Y MEDIO AMBIENTE
}

\author{
ANNA BATET Figueras \\ Máster en Antropología Médica y Salud Global \\ Universitat Rovira i Virgili \\ annabfigueras@gmail.com
}

RESUMEN: Las formas de producción en la actividad agrícola pueden convertir productos «sanos» desde la perspectiva nutricional en «peligrosos» desde la perspectiva de la salud. El presente artículo se centra en la percepción del riesgo para la salud de los agroquímicos, un riesgo que suscita nuevos estudios sobre la percepción de la calidad alimentaria. El ámbito de estudio se sitúa en la costa de Oaxaca (México) con la voluntad de examinar cómo las percepciones de los agricultores sobre la contaminación química, en el contexto socioeconómico y cultural particular, intervienen en las representaciones sociales del riesgo y la seguridad alimentaria. En este sentido, los conceptos «flojo» (vago) y «débil» constituyen factores socioculturales que condicionan la percepción del riesgo de los agroquímicos usados en el campo, y, por ende, el uso de medidas de protección o el itinerario terapéutico en caso de intoxicación. Se analiza también cómo estos discursos sobre la percepción del riesgo influyen en las prácticas agrícolas y en la presencia de alternativas al modelo agrícola industrial.

PALABRAS CLAVE: percepción del riesgo; seguridad alimentaria; agroquímicos; Oaxaca. 
ABSTRACT: Forms of production in agricultural activity can turn "healthy" products from the nutritional perspective into "dangerous" products from the perspective of health. This paper focuses on the perception of food risk in relation to the use of agrochemicals in agriculture, a risk to health that motivates new studies on the perception of food quality. The field of study is located on the coast of Oaxaca (Mexico) and the objective is to examine how farmers' perceptions of chemical contamination, in the particular socioeconomic and cultural context, contribute to social representations of risk and food security. In this sense, the "flojo" (lazy) and "débil" (weak) concepts are sociocultural factors that condition the perception of risk associated with agrochemicals used in the field, hence the use of protective measures or therapy in case of intoxication. The paper also analyses how these discourses on the perception of risk influence agricultural practices and the presence of alternatives to the industrial agricultural model.

KEYWORDS: risk perception; food security; agrochemicals; Oaxaca. 


\section{Introducción}

El sistema alimentario actual está lleno de contradicciones: encontramos personas que pasan hambre ( 815 millones) en un mundo donde se producen alimentos para más de 12.000 millones de personas (Vivas, 2014). Según los informes de organismos como la FAO, la OPS, la OMS y la IAASTD ${ }^{1}$, la seguridad alimentaria es motivo de preocupación a nivel internacional y plantea nuevos retos para la agenda 2030 de desarrollo sostenible de la $\mathrm{ONU}^{2}$. Hasta ahora, la FAO ha centrado el análisis de la seguridad alimentaria en la cuestión de la accesibilidad y la malnutrición, si bien recientemente ha incorporado nuevos argumentos en sus informes que relacionan la seguridad alimentaria con el cambio climático y los conflictos sociopolíticos (FAO et al., 2017). En cambio, la cuestión de la calidad alimentaria parece que no avanza al mismo ritmo ante un sistema agrícola mundial que depende del petróleo, adapta las semillas a los intereses del mercado y provoca la dependencia de las variedades agrícolas a un uso intensivo de agrotóxicos y fertilizantes químicos. La acepción de food safety, el consumo de alimentos libres de tóxicos, está estrechamente relacionada con nociones como las de riesgo y sufrimiento ambiental mediante la correlación entre la contaminación que proviene del ambiente y las formas de producción (riesgo ambiental), y la que se desplaza dentro del cuerpo a través de la alimentación (riesgo alimentario). La contaminación química puede tomar diferentes significados en función del contexto sociocultural, y a pesar de que la noción de riesgo no es específica de un contexto determinado, sí lo son sus manifestaciones y percepciones (Gracia, 2002).

En el presente artículo, los contaminantes químicos (pesticidas, fertilizantes, hormonas de crecimiento, etc.) toman el protagonismo en la percepción social e individual del riesgo alimentario. Centrándome en el uso de agroquímicos en la actividad agrícola, me pregunto qué consecuencias colaterales tienen sobre la salud de las personas y el medio am-

1 Acrónimos de Food and Agriculture Organization (FAO), Organización Panamericana de la Salud (OPS), Organización Mundial de la Salud (OMS), e International assessment of agricultural knowledge, science and technology for development (IAASTD).

2 Para más información, véase <https:/www.un.org/sustainabledevelopment/es/2015/09/la-asamblea-general-adopta-la-agenda-2030-para-el-desarrollo-sostenible/> (último acceso: 20-5-2018). 
biente, con el objetivo principal de conocer de qué manera este modelo de producción agrícola intensivo influye en la percepción del riesgo para la salud de los agricultores, y cómo esta percepción del riesgo se articula en discursos y prácticas a favor de una agricultura más sostenible y respetuosa con el medio ambiente.

El escenario de investigación se sitúa en el estado de Oaxaca, concretamente en la franja costera del municipio de Villa de Tututepec de Melchor Ocampo (en adelante, Tututepec), incluyendo las comunidades de Santa Rosa de Lima, San José del Progreso, Río Grande, El Renacimiento, San Miguel, Playa Vieja, Camalotillo, Charco Redondo, Chacalapa, La Luz, San Vicente, Santa Cruz y Santiago Jocotepec. El estado de Oaxaca ocupa el segundo lugar en el ranking de los más pobres del país, y según los indicadores de bienestar de la $\mathrm{OCDE}^{3}$, ocupa el penúltimo lugar en accesibilidad a servicios, en ingresos y en educación. El tercer municipio más grande del estado y el más grande de la región costa es Tututepec, que se encuentra clasificado con un grado de marginación alta y un grado de retraso municipal medio ${ }^{4}$ por la Secretaría de Desarrollo Social (SEDESOL) de México. Se calcula que un 51,2\% de la población de Tututepec tiene carencias alimentarias por acceso a los alimentos (Ceciproc y Ecosta, 2018; Coneval, 2015; INEGI, 2015).

Sabemos que las causas de la inseguridad alimentaria en México no son debidas a la insuficiencia de alimentos producidos , hecho por el cual es necesario abordar el problema desde una perspectiva multidimensional que actúe sobre el acceso a los recursos, los activos y las capacidades, sobre la oferta, sobre la disponibilidad alimentaria y sobre la forma de producir los alimentos, entre otros. Por otra parte, sabemos que el logro de la suficiencia alimentaria no asegura una alimentación sana y nutritiva, ni alimentos de buena calidad e inocuos. Las noticias, estudios y propuestas reclamando una mayor inocuidad de los alimentos van tomando protagonismo en el país (Arellano et alii, 2009; Díaz-Barriga, 1996; García

\footnotetext{
3 Véase: <https://www.oecdregionalwellbeing.org/ME20.html> (último acceso: 20-5-2018).

4 Este índice es la media ponderada de indicadores de carencias sociales: educación, salud, acceso a servicios básicos y espacios de la vivienda. Los datos son de 2010 y han sido extraídos de: <http:// www.microrregiones.gob.mx/catloc/Default. aspx?buscar $=1 \&$ tipo $=$ nombre\&campo=mun\&valor $=-$ tututepec\&varent=> (último acceso: 20-5-2018).
} 
Hernández et alii, 2018; Karam et alii, 2004; Ortega-Ceseña et alii, 1994), sobre todo si tenemos en cuenta que en México se utilizan plaguicidas altamente peligrosos que están prohibidos en otros países. La OMS ha estimado que se producen entre 2 y 5 millones de casos de intoxicación por pesticidas y que aproximadamente generan 40.000 muertes al año, y pese a la evidencia de los impactos negativos sobre la salud humana y el medio ambiente, el uso de plaguicidas está aumentando a escala mundial con una tasa de crecimiento entre el 4 y el 5,4\% anual (IAASTD, 2009). La Dirección General de Epidemiología de la Secretaría de Salud de México (2013) notificaba que había habido 67.711 casos de intoxicación por plaguicidas en todo el país en el período comprendido entre los años 1995 y 2012 , principalmente en los grupos de edad productiva (60\%) y de sexo masculino $(71,02 \%)$, lo que nos indica que esta exposición constituye un riesgo elevado en la actividad laboral. En lo que va de año, en Oaxaca, uno de los estados que en los últimos años ha presentado una tendencia al alza en el uso de agroquímicos, ya se han reportado 14 intoxicaciones por plaguicidas 5 .

Con la modernización agrícola iniciada en la década de los años cuarenta del siglo pasado, se pretendía lograr el crecimiento y el desarrollo agrario aumentando la producción de alimentos, introduciendo la mecanización, la irrigación, los fertilizantes químicos, las semillas de alto rendimiento y los cultivos transgénicos, entre otros. La revolución verde (RV) se gestó en México ${ }^{6}$ y desde allí se dio inicio a la importación del «paquete tecnológico» ${ }^{7}$ que debería aumentar la producción y productividad cerealera de los países pobres mediante el uso intensivo de la tierra (Elvia, 1991). Quienes enfatizan las ventajas de la RV se basan en la neutralidad de la ciencia y la tecnología y apelan al incremento de la producción para

5 Véase: <https://www.gob.mx/cms/uploads/attachment/file/447667/semil.pdf> (último acceso: 273-2019).

6 La CIMMYT (<https:/www.cimmyt.org/>) y la Fundación Rockefeller financiaron programas de investigación genética para producir variedades de alto rendimiento, entre otros. Actualmente se dedican a la investigación, educación y al desarrollo de planes y programas de desarrollo agrícola en general.

7 Un paquete tecnológico incluye las semillas mejoradas y todos los agroquímicos asociados (herbicida, insecticida, fertilizante, etc.) que necesitará el agricultor para producir con el «máximo rendimiento», rendimiento que a la larga se transforma en un estancamiento o incluso en un decrecimiento de la producción. A eso hay que añadirle el efecto de contaminación y degradación del ecosistema. 
solucionar los problemas del hambre y del desarrollo en los países rurales (Mellor, 1989, en Elvia, 1991). Pero sabemos que la tecnología no puede eliminar por sí sola el hambre ni la pobreza en el campo, y que la RV, influida por la teoría de la modernización de corte liberal, favoreció principalmente a terratenientes y a grandes empresarios agrícolas, quienes acapararon los recursos físicos, tecnológicos y financieros.

Actualmente, la importante influencia política y económica del agro business no solo ejerce presión en el ámbito normativo y político, sino que pone trabas a la investigación y promoción de alternativas agroalimentarias, menosprecia los resultados de las investigaciones sobre los efectos nocivos de los plaguicidas y debilita la evaluación normativa de los riesgos (IAASTD, 2009). De este modo, hemos puesto el rumbo hacia un modelo alimentario global extremadamente vulnerable a las crisis económicas, ecológicas y sociales, donde la agricultura se erige como una de las dos principales responsables de la pérdida de agrobiodiversidad y contribuye de manera significativa al cambio climático (GRAIN, 2011; Montagut y Vivas, 2009). Hoy en día disponemos de una gran cantidad de argumentos científicos sobre la sostenibilidad de las prácticas agroecológicas tradicionales para afrontar los retos vinculados con la seguridad alimentaria, pero estas soluciones son extremadamente difíciles de implementar por la amenaza que representan a las ventas y rentabilidad de la potente industria alimentaria.

Con el presente estudio se pretende, por un lado, hacer llegar la voz de los agricultores sobre la percepción de la seguridad alimentaria y demostrar cómo los discursos sobre la percepción del riesgo de los plaguicidas se articulan, o no, con iniciativas a favor de modelos de producción agrícola más sostenibles y saludables, y, por el otro, contribuir en los debates críticos sobre los manufactured risks, la distribución de los conflictos socioecológicos y las formas de producción capitalistas en el marco de la justicia ambiental. En el campo de la antropología médica, la contribución del estudio se enmarcaría en los análisis antropológicos sobre salud, desarrollo y medio ambiente, tomando el concepto de riesgo como eje teórico central. 


\section{Metodología}

La metodología usada en el presente trabajo es la cualitativa de base etnográfica y las técnicas utilizadas son la entrevista semiestructurada y la observación directa. Las unidades de análisis son las parcelas agrícolas y los actores sociales dentro de esta unidad de análisis son, en primer lugar, los agricultores que trabajan directamente en el campo, y en segundo lugar, la familia y el resto de actores vinculados con la actividad agrícola, como los técnicos agropecuarios, los representantes comunales/ejidales ${ }^{8}$ de los terrenos agrícolas y las asociaciones civiles que promueven la defensa y la recuperación del patrimonio natural y sociocultural. El número total de entrevistas realizadas ha sido de 43 (20 agricultores, 15 autoridades y 8 miembros de asociaciones civiles) y se han llevado a cabo entre enero y abril de 2018. Casi todos los contactos y las citas con los informantes (a excepción de 6 casos) son facilitados por ECOSTA?.

Los participantes del proyecto, mayoritariamente hombres de edades comprendidas entre los 36 y los 55 años, se distinguen en tres grupos: a) agricultores, que son el objeto de estudio; $b$ ) autoridades técnicas/médicas/políticas ${ }^{10}$, con el fin de obtener una visión desde el sector médico, técnico o político en relación con el tema de estudio; c) activistas/asociaciones civiles, para conocer los discursos de los movimientos sociales y las propuestas de alternativas al modelo agroalimentario. La distinción de los tres perfiles de entrevistados responde al reconocimiento de la multiplicidad de actores que intervienen en la estructura social y de significado, incorporando el enfoque relacional en el análisis etnográfico (Menéndez, 2009). Cabe mencionar que algunos informantes cumplen con dos de los roles mencionados a la vez, pero la clasificación se efectúa en función del rol predominante que asume el entrevistado en el discurso.

\footnotetext{
8 En Oaxaca, el $80 \%$ del territorio es de propiedad comunal, es decir, la propiedad de la tierra es mayoritariamente social. La autoridad agraria que gestiona la tierra es el Comisariado de Bienes Comunales o Comisariado Ejidal, según el caso.

9 Ecosta Yutu Cuii, SSS, fundada en 2004, es una entidad que se dedica a la concienciación ambiental y a la promoción de proyectos de desarrollo local, poniendo especial énfasis en el fomento de la agricultura orgánica.

10 Por autoridades políticas se toman los representantes municipales o de la propiedad comunal.
} 


\section{El riesgo alimentario ${ }^{11}$ y el riesgo ambiental}

Actualmente, el sector agroalimentario mundial se apoya sobre una estructura productiva dependiente de los agroquímicos, que nos emplazan en una situación de vulnerabilidad e incertidumbre permanentes tanto para los consumidores como para los productores. ¿Hay riesgos aceptables o razonables? Las Naciones Unidas recomiendan dos definiciones divergentes para evaluar la toxicidad de los productos químicos: una centrada en la probabilidad, entendiendo el riesgo como un concepto estadístico, y la otra centrada en propiedades de utilidad, donde el beneficio entra en la ecuación del riesgo tratando la seguridad como una medida de la aceptabilidad de un cierto grado de riesgo (Douglas, 1996). Como meta de los Objetivos de Desarrollo Sostenible (ODS) ${ }^{12}$, la minimización del riesgo de los plaguicidas se aborda desde la perspectiva del riesgo como «cálculo de probabilidades» sujeto a los beneficios económicos. Esta es una de las críticas que presenta Hilal Ever en el análisis de la cuestión del riesgo, abordándolo desde el derecho a la alimentación, derecho que no debería poner en peligro la salud humana ni el medio ambiente (ONU A/HRC/34/48, 2017).

La teoría cultural de Douglas y Wildavsky (1983) parte de la idea de que el riesgo es una construcción social, donde cada cultura construye y representa sus riesgos en función de un sistema de valores y creencias, y en función de la posición social y personal en el seno de la sociedad (Gracia, 2004). En cambio, el enfoque teórico defendido por Beck (1998; 2002), Giddens (1994) o Bauman (2001) sobre la sociedad del riesgo pone el énfasis en los aspectos (macro) estructurales de la organización económica y política de las sociedades modernas y en los manufactured risks que las caracterizan (Gracia, 2004). Bajo este paradigma, el sistema productivo y las aplicaciones tecnológicas se convierten en las causas principales de los efectos negativos sobre el medio ambiente y la salud, y las percepcio-

11 Según Gracia (2004), el 20 de marzo de 1996 se puede considerar la fecha que marca el inicio de la era del riesgo alimentario (en términos de inocuidad y calidad alimentaria) debido a la enfermedad de las vacas locas. Por otro lado, este episodio coincide con las medidas tomadas por la UE para hacer frente a los efectos sobre el medio ambiente de la agricultura intensiva (disminuir uso de pesticidas, cultivo de especies locales de calidad y aumento de los controles sanitarios, entre otros).

12 Véase: <https://www.un.org/sustainabledevelopment/es/objetivos-de-desarrollo-sostenible/> (último acceso: 28-5-2018). 
nes sobre los riesgos se deben más a una respuesta lógica y racional de las incertidumbres advertidas, tanto individualmente como colectivamente, que no a una estructura subyacente sobre cómo y por qué unas situaciones son susceptibles de problematizarse y otras no. Es así como el cuestionamiento de las instituciones públicas y científicas sobre los riesgos generados por la tecnificación en el ámbito agroalimentario favorece el establecimiento de nuevos debates sobre las identidades sociales y morales de los pueblos, que se manifiestan en movimientos sociales de diversa índole (Gracia, 2004).

Entrando en el desarrollo conceptual de los riesgos alimentarios, estos los podemos agrupar en tres bloques: los riesgos relacionados con las formas de consumo, los riesgos sanitarios, higiénicos y bacteriológicos de los alimentos (los que recientemente tienen más presencia en los medios de comunicación) y los riesgos relacionados con las formas de producción, que son el objeto de estudio principal del presente trabajo. Según Menéndez (2009), el pensamiento hegemónico sitúa los riesgos y los factores de riesgo en el centro, dejando de lado el contexto y el medio ambiente y responsabilizando únicamente al individuo. Asimismo, Reith (2004) considera que la utilidad del cálculo del riesgo tiene limitaciones, ya que individualiza la gestión de riesgos que son colectivos. Este enfoque reduce el riesgo pensándolo en términos de estilo de vida y desplazándolo hacia dentro del cuerpo, haciendo aparecer una nueva inquietud: «el riesgo corporal». La contaminación «química» de los alimentos puede ser debida a la presencia de restos de productos fitosanitarios aplicados sistemáticamente en la producción, a la falta de controles ambientales o a la utilización inadecuada de ciertas tecnologías o productos agroquímicos. En este sentido, hay autores que investigan los riesgos para la salud de esta contaminación química interna desde diferentes perspectivas epistemológicas, teóricas y metodológicas (Begueria, 2016; Larrea-Killinger et alii, 2016; Porta et alii, 2002).

Los riesgos relacionados con las formas de producción de los alimentos también tienen efectos sobre el medio ambiente, y desde mi punto de vista, los riesgos ambientales y los riesgos para la salud humana deben ser considerados desde una perspectiva holística e integradora . Tradi- 
cionalmente los riesgos ambientales han sido asociados al medio exterior, y a la hora de vincular la toxicidad ambiental con nuestro medio «interior», surge la incertidumbre con sus matices en función del contexto particular, como muestran los trabajos etnográficos de Auyero y Swistun (2007), Freitas et alii (2009) y Little (2012). Según Beck, el riesgo ambiental está latente, conlleva efectos socioambientales negativos y es un fenómeno global que se debe analizar y discutir localmente. Cuando este se materializa en desastres ambientales, se manifiestan las dimensiones de vulnerabilidad social de la población y del entorno en el que viven (Alfie, 2017). En este artículo, cuando hablo específicamente de riesgo ambiental me refiero a los efectos indirectos o a largo plazo de la contaminación ambiental sobre la salud humana.

Otra aproximación teórica que considero interesante es la procedente de la ecología política, al considerar que todas las relaciones materiales de intercambio social entre las personas implican necesariamente intercambios dentro de las relaciones naturales (Busqueta et al., 2017). De este modo, la teoría del metabolismo socioecológico (Küster, 2016; Toledo, 2013) se convertiría en un ejemplo de superación de la dicotomía sociedad-naturaleza, ya que nos permite analizar los sistemas agroalimentarios considerando tanto las ciencias sociales como las ciencias naturales. Por otra parte, el campo de la ecología política se está desplazando más allá de los contextos rurales locales hasta abarcar los conflictos ecológicos distributivos, que los economistas ortodoxos disfrazan bajo el nombre de «externalidades». Estudios de los conflictos socioecológicos como la degradación de la tierra, la biopiratería, el extractivismo o la exposición a diferentes formas de riesgos ambientales y laborales pertenecen tanto a la ecología política como a los movimientos de justicia ambiental (Flocks y Monaghan, 2003; Martínez Alier, 2006; Saxton, 2015; Ceccam, 2017; Ceccam y Bröt, 2015).

Desde la perspectiva de la antropología médica crítica, considero interesante el trabajo de Menéndez (1981) sobre los sistemas de transacciones en el ámbito mexicano, ya que describe las formas de explotación, dominación y hegemonía de las élites sobre las clases subalternas y se convierte en una herramienta de denuncia de gran valor frente al sistema hegemónico que condiciona el marco de necesidades de los agricultores, 
determinando la dimensión económica, política e ideológica de su modo de vida (Martínez-Hernáez, 2011). En este sentido, podemos entender los pesticidas como una forma de dominación de las élites de la agroindustria sobre los campesinos de la costa de Oaxaca.

\section{El proceso de modernización agraria: el uso de plaguicidas en México}

El municipio de Tututepec tiene una larga historia agrícola que ha sufrido cambios importantes en las últimas décadas, causando impactos sobre los recursos naturales, el material genético, las prácticas de cultivo y el consumo alimentario de las comunidades (Lara y Reyes, 2010). Actualmente, los principales productos que se cultivan y comercializan en la zona son la papaya, el limón, el cacahuete, el coco, el plátano, el café y el sésamo, yendo en aumento el cultivo de sandía. El trabajo de campo se realiza en las comunidades rurales ubicadas en la planicie, donde se ha extendido principalmente la agricultura comercial y donde se han aplicado en mayor medida fertilizantes y agroquímicos, algunos de los cuales son considerados altamente peligrosos (Bejarano, 2017; Díaz-Barriga, 1996; Karam et alii, 2004). La agricultura, que representa el 55\% de los sectores productivos del municipio, es una agricultura principalmente industrial que se combina con algunos elementos de la agricultura tradicional, como la roza-tumba-quema $a^{13}$ y la milpa ${ }^{14}$.

La agricultura industrial en la zona de estudio empieza a tomar fuerza en la década de los setenta, a partir de los monocultivos de algodón, que incorporaron la visión mercantilista de la actividad agrícola. Como es sabido, el objetivo de la agricultura industrial es la mejora del rendimiento y la eficiencia del sistema productivo, y esto explica la promoción y difusión de los «paquetes tecnológicos» en México mediante los diferentes programas gubernamentales, conllevando la pérdida de semillas

13 La RTQ es una concepción productiva que se basaba en un período de barbecho generoso, favorecido por el carácter de rotación y mudanza de la actividad agrícola y por la gran variedad de usos del suelo en una misma finca.

14 Es un sistema de producción diversificada compuesto principalmente de maíz, calabaza, frijol y chile. 
nativas y el uso continuado de plaguicidas y fertilizantes. Cuando dejó de ser rentable el cultivo de algodón, la costa de Tututepec continuó apostando por los monocultivos transformándose primero en zona limonera, cultivo todavía principal a día de hoy, y en zona papayera posteriormente. El cultivo de la papaya, sin embargo, implica un uso de agroquímicos, «tan peligroso como el uso de agroquímicos que se realizaba en los años ochenta durante la siembra del algodón» (RRM, ingeniero agrónomo), según la opinión de algunos de los informantes.

El impulso de la revolución verde, combinado con los Gobiernos neoliberales que ha habido en las últimas décadas en México, ha favorecido el desarrollo de un conjunto de políticas públicas para apoyar a la agroindustria, apostando por la agricultura de exportación y dejando de lado a los pequeños agricultores. Los hechos más destacados que han favorecido el actual escenario mexicano son, por un lado, la entrada en vigor del Tratado de Libre Comercio con América del Norte y Canadá (TLCAN), que provocó cambios ${ }^{15}$ en los mecanismos de fijación de los precios internos afectando la producción agrícola nacional (Montagut y Vivas, 2009), y condicionó el marco normativo de plaguicidas. Por otro lado, la crisis financiera, energética y alimentaria de 2008 tuvo un impacto negativo sobre la seguridad alimentaria mundial, haciendo que en México se desencadenaran un conjunto de hechos trascendentales que modificarían el escenario de la política federal sobre la seguridad alimentaria: el programa nacional México sin hambre 2014-2018 (Urquía-Fernández, 2014).

Según datos del Comité estatal de Sanidad Vegetal (CESVI), de los 2.500 productores agrícolas que hay en el municipio de Tututepec, un $40 \%$ aplicarían agroquímicos de manera muy intensiva durante todo el año y en varias ocasiones; otro $40 \%$ aplicarían agroquímicos de manera moderada, y un $20 \%$ aplicarían pesticidas una vez al año o utilizarían aceites y productos de «bajo impacto» ${ }^{17}$. Dentro de este último grupo se incluyen los que hacen agricultura orgánica o natural.

15 Para mayor información, véanse: <http://ceccam.org/node/2265>; $\langle$ http://ceccam.org/node/403>; $<$ http://ceccam.org/node/366> (última consulta: 18-5-2018).

16 Véase: <http://www.plataformacelac.org/politica/7> (última consulta: 18-5-2018).

17 Estos datos provienen de una conversación informal con los técnicos del CESVI, por lo tanto, son aproximados, no oficiales y están extraídos de su base de datos a petición personal para la presente investigación. 
La autorización gubernamental para la venta y uso de plaguicidas en México la efectúa la Comisión Federal para la Protección contra Riesgos Sanitarios (COFEPRIS) a través de un registro sanitario único que incluye la opinión técnica de la Secretaría de Medio Ambiente y Recursos Naturales y de la Secretaría de Agricultura, Ganadería, Desarrollo Rural y Pesca, tal como especifica el reglamento para el registro de plaguicidas, fertilizantes y sustancias tóxicas (R-PLAFEST, 2014). A partir de los estudios toxicológicos, ecotoxicológicos, de afectación ambiental y de efectividad biológica de un plaguicida, requeridos por el reglamento PLAFEST, parecería que la evaluación realizada por los organismos del estado garantiza la seguridad a la hora de incluir un plaguicida en el catálogo de plaguicidas autorizados ${ }^{18}$. Pero la realidad es que no se puede garantizar que no haya riesgos inaceptables para la salud y el ambiente, ya que existe un vacío legal en la evaluación de riesgos (no es una obligación legal según el mismo reglamento), y consecuentemente no se aplica el principio precautorio en la negación o revocación de un registro.

Además, los plaguicidas autorizados antes de 2005 tienen una vigencia indeterminada y a los que se registran a partir de esta fecha no se les requiere información adicional para actualizar el registro, que a efectos prácticos se traduce en una mayoría de plaguicidas autorizados $(80,7 \%)$ con una vigencia indeterminada en el país (Bejarano et alii, 2017). En resumen, ante las vicisitudes del procedimiento legal descrito más el plazo limitado para revisar la información proporcionada por la empresa agroquímica, no es de extrañar que en México haya un gran número de plaguicidas altamente peligrosos (PAP) autorizados o prohibidos en otros países. Por ejemplo, en el catálogo de plaguicidas de la COFEPRIS de 2016 , encontramos que 183 ingredientes activos son altamente peligrosos, de los cuales el 34,43\% presentan elevados efectos tóxicos agudos en la salud humana (Bejarano et alii, 2017), y de estos, 140 están prohibidos o no autorizados en otros países.

18 El catálogo de la COFEPRIS contiene los plaguicidas registrados y autorizados para la importación, comercialización y uso en el territorio mexicano. 


\section{La representación social del riesgo de los agrotóxicos}

\subsection{Los discursos}

Los discursos de todos los entrevistados se hallan atrapados en un círculo vicioso trazado por la presión del mercado, la necesidad de insumos para producir, el incremento de plagas y la disminución de la productividad del suelo. Parece no existir ninguna alternativa al modelo agrícola industrial.

Usamos la agricultura convencional porque todos queremos cantidad, producir más. A los árboles los saturamos de fertilizantes (OCS, agente municipal de Santa Rosa de Lima).

A fuerza hay que usar fertilizante, si no, no hay rendimiento (MMR, Comisariado de Bienes Comunales).

Lo que pasa es el tiempo y el dinero. Con 1 litro de faena (herbicida) aventajo bastante, lo que no avanzo con dos piones (JLT, agricultor).

En general, el riesgo de los agroquímicos se percibe en forma de intoxicación aguda, situando al margen los malestares y las enfermedades crónicas, e incluso las muertes atribuidas al agrotóxico. La desinformación sobre los peligros de los químicos genera incertidumbre, pero el enfoque comercial de la agricultura se encuentra tan arraigado que los discursos a favor de la reducción de agroquímicos en el campo se contradicen con la necesidad de mantener «limpios» los campos (factores socioculturales), o son descartados debido a las dificultades entramadas por los sectores que operan a nivel macroestructural.

Centrándonos en el tema de estudio, podemos observar la situación de ambivalencia en la que nos posicionan los pesticidas cuando nos situamos en la escala de riesgo/beneficio, generándonos sentimientos opuestos y contradictorios simultáneamente, tanto en el ámbito ambiental como en el de la salud de la persona (Widger, 2014). En general, se aceptan como «productos peligrosos» con un amplio abanico de afecciones sobre la salud y el medio, pero también como promotores de la agricultura y el desarrollo social y económico. De este modo, la mayoría de informantes ${ }^{19}$

19 Cabe mencionar que, en el perfil de autoridades, la mitad de las personas entrevistadas también trabajan el campo como agricultores. 
que trabajan el campo $(81,5 \%)$ continúan utilizando agroquímicos a pesar de la percepción elevada de riesgo.

Para mí sería un mal necesario. Te está ayudando, pero a la larga te lo va a cobrar (MMR, Comisariado de Bienes Comunales).

Yo oigo que hay mucho cáncer y paso a pensar que puede ser por tanto químico que se le aplica a la fruta (JLT, agricultor).

Según Beck (1998), la expansión de los riesgos va de la mano del desarrollo capitalista, que, por un lado, hace aumentar la productividad, y, por el otro, provoca enfermedades. En la sociedad actual, la necesidad inmediata creada por el sistema capitalista compite con el riesgo conocido, y la apuesta entre la riqueza que se percibe y los riesgos que son imperceptibles será ganada por la primera. Según el autor, la paradoja es que precisamente por eso (invisibilidad), los riesgos acaban ganando la apuesta (ibid.: 63) y el aprovechamiento económico de estos origina situaciones de peligro y vulnerabilidad social y ambiental.

El riesgo no lo conocemos, pues nosotros no lo vemos, y los beneficios sí. El perjuicio, como uno no lo ve, no sabe ni de qué uno se enfermó (PAP, Comisariado de Bienes Comunales).

La agricultura orgánica tiene beneficios que no los vamos a ver y a la larga. Si hubiera un mercado orgánico, estaría bien para notar un beneficio en el bolsillo, porque en la salud no lo vamos a notar (MMR, Comisariado de Bienes Comunales).

Más allá de estas generalidades sobre la percepción del riesgo de los agroquímicos, el análisis de los discursos nos permite conocer cuál es la representación social del riesgo para los tres perfiles de informantes. Empezando por los agricultores, la incertidumbre y la confusión manifestada hacia la toxicidad de los agroquímicos es destacable y a menudo admitida directamente. Esta se debe principalmente a la falta de información sobre los riesgos y peligros asociados a su uso: «No tenemos un estudio, no sabemos en qué nos estará haciendo mal» (MEB, presidente de la Junta Local de Sanidad Vegetal). La desinformación es especialmente acusada en el grupo de agricultores, disminuyendo o incluso desapareciendo en los grupos de autoridades y activistas. Si además añadimos 
la falta de «hábito» en el uso de equipos de protección individual o EPI (el 71\% de los agricultores no los usan), nos emplazamos en una situación de vulnerabilidad social notable ${ }^{20}$. Esta incertidumbre sobre la peligrosidad del producto agroquímico en sí se traslada e influye en los factores de exposición, en las medidas de mitigación adoptadas y en el itinerario terapéutico escogido.

Vemos que para los agricultores es destacable la utilización del olor y las experiencias de intoxicación de otros compañeros como medios para seleccionar y clasificar los riesgos de un agroquímico. Solo para aquellos plaguicidas pestosos o fuertes se utilizan medidas de protección (o es más probable que se utilicen): pañuelos para cubrirse la nariz y la boca, gafas, guantes, y en algunos casos lonita para la espalda, pantalones y camisa de manga larga y botas. Cabe mencionar que la mayoría rechazan los EPIS por el calor que hace en la región y porque muchos productos no son considerados peligrosos, como los fertilizantes, la mayoría de los herbicidas y en general todos aquellos productos que no dan olor.

El rasgo más destacable del análisis de los discursos es la presencia de los conceptos flojo (vago) y débil, entendidos como factores socioculturales que condicionan la percepción social del riesgo de los agroquímicos. Ser flojo se relaciona, por un lado, con la presencia de monte o maleza en la parcela, que a su vez se vincula con la presencia de plagas y con el bajo rendimiento del cultivo. Tener «limpio» el campo es sinónimo de ser trabajador y si tienes maleza significa que eres vago, y esto es motivo de escarnio.

Si tú tienes limpio el campo, eres muy trabajador, pero si tienes monte, eres flojo, es terreno de viuda se dice. Se van a burlar y van a decir que este limón es de viuda (HFC, activista).

Por otro lado, flojo también se relaciona con la sustitución de la manera tradicional de trabajar el campo: «Ahora el campesino se volvió flojo, antes uno no se despegaba del machete» (JRA, agricultor). Esta segunda

20 La NOM-003-STPS-1999 es la norma federal que establece las condiciones de seguridad e higiene para prevenir los riesgos a los que están expuestos los trabajadores agrícolas, y obliga, entre otras, tanto a los patrones como a los agricultores a utilizar EPI. En la práctica, las bombas de fumigar se encuentran en mal estado, pierden líquido y entra en contacto con la piel del agricultor, que raramente va protegido con un equipo de protección adecuado. 
acepción es usada principalmente por los agricultores orgánicos, que no han sustituido el machete por el líquido ${ }^{21}$.

El concepto débil está relacionado con la construcción de la identidad masculina ${ }^{22}$ y la creencia en la inmunidad subjetiva ante el daño físico o la enfermedad a la hora de afrontar el riesgo de los agroquímicos: solo se intoxican los que son débiles. Algunos informantes hablan de aquellos que «dicen que son fuertes y no les hace nada» o los que son «débiles y luego empiezan a vomitar» (PAP, Comisariado de Bienes Comunales). Entonces, tanto flojo como débil tienen connotaciones peyorativas que desacreditan, estigmatizan e influyen en la percepción y la exposición a los riesgos de los agroquímicos utilizados en la actividad productiva. No es de extrañar, entonces, que en caso de intoxicación muchos agricultores se automediquen (antihistamínicos), se «autorregulen» descansando unos días antes de volver a fumigar o usen el «saber popular» para prevenir los efectos de la intoxicación y evitar los casos graves que requieren la atención del servicio médico especializado.

El grupo de autoridades técnicas, médicas y políticas centra los discursos casi exclusivamente en la sintomatología aguda asociada a los agroquímicos (mareos, vómitos y pérdida de conciencia) y apoyada por la evidencia científica, sin entrar en el terreno de la cronicidad manifestada en malestares y enfermedades diversas ${ }^{23}$. Quizá, por este motivo, los ingenieros agrónomos y los médicos ponen más énfasis en el riesgo relacionado con las malas prácticas y la incorrecta manipulación de los agroquímicos que en la peligrosidad del producto en sí. Como perciben el ámbito productivo como la principal vía de exposición al riesgo de los agroquímicos (73\% de los entrevistados), consideran que las técnicas de mitigación que deberían potenciarse son las formaciones sobre las medidas de seguridad y sobre la manipulación y el buen uso de los agro-

\footnotetext{
21 Durante las entrevistas se utiliza el término líquido para referirse al agroquímico.

22 Un estudio interesante en esta línea es el de Calvario Parra (2007).

23 Hay que especificar que los médicos entrevistados corresponden al nivel de atención primaria, y esto explicaría los pocos casos de intoxicaciones que han tratado personalmente e influiría en la percepción del riesgo y en el conocimiento de la etiología relacionada con los agroquímicos. Por ejemplo, la enfermera de Santa Rosa no me sabe decir cuáles son las vías de exposición más habituales en caso de intoxicación por plaguicidas porque dice que no han tenido nunca un paciente con estas características.
} 
químicos; el respeto y seguimiento escrupuloso del método de aplicación recomendado por el ingeniero agrónomo; el uso de EPI, y la atención en las medidas de higiene después de aplicar los plaguicidas.

Sería en el caso los activistas y/o miembros de asociaciones civiles donde podemos ver la conjugación más completa de los diferentes elementos presentes en los discursos de los informantes. El riesgo para la salud se relaciona tanto con las intoxicaciones agudas como con el incremento de enfermedades que ha habido en la zona en los últimos años ${ }^{24}$, percibiendo el riesgo más allá del momento presente. La asociación entre riesgo, salud y calidad alimentaria incluye el ámbito productivo de la actividad agrícola, pero también el consumo alimentario y el estado de degradación del medio ambiente. La vinculación entre salud ambiental y salud humana toma especialmente relevancia en el grupo de activistas, aunque los agricultores también diferencian y clasifican los productos químicos entre los que perjudican la tierra (menor fertilidad, salinización y degradación del suelo) y los que no. En algunos informantes, los efectos del medio son vistos de forma más integral, como la falta de agua, la afectación de la cadena trófica o la contaminación por los residuos asociados al uso de agroquímicos, y en otros, la salud ambiental se subordina a las necesidades del mercado (la productividad del suelo) y se desliga de la salud humana.

\subsection{Las evidencias científicas}

De los 13 plaguicidas más utilizados por los informantes todos están prohibidos en otros países y clasificados por los organismos internacionales como altamente peligrosos, a excepción del tordon y el esteron. El gramo$x^{x} e^{25}$, el herbicida más utilizado y percibido por los informantes como de «no mucho riesgo», está prohibido en 38 países y clasificado según la normativa europea en la categoría I-II: toxicidad aguda o mortalidad en caso de inhalación (Parlamento europeo y Consejo de la UE, 2008)etiquetado y

24 Principalmente serían las relacionadas con el síndrome metabólico (obesidad, diabetes, hipertensión) y el cáncer.

25 Los efectos del paraquat en la salud humana son alteraciones del tracto gastrointestinal, riñón, hígado y corazón, entre otras. Para más información: https://espanol.epa.gov/sites/production-es/ files/2015-09/documents/spch12.pdf (última consulta: 9-6-2018). 
envasado de sustancias y mezclas, y por el que se modifican y derogan las Directivas 67/548/CEE y 1999/45/CE y se modifica el Reglamento (CE.

Los discursos sobre la percepción del riesgo para la salud se intercalan con las experiencias propias, de familiares y/o de conocidos sobre malestares y enfermedades que son consecuencia de la interacción entre los agroquímicos y el cuerpo humano. En los tres grupos de informantes encontramos experiencias cercanas de afectación a la salud vinculadas a los agroquímicos, ya sean protagonizadas en primera persona, por familiares o conocidos. Los grupos que más experiencias comparten son los agricultores, donde la mitad de los entrevistados conocen casos cercanos, y las autoridades, donde todos los que se dedican al trabajo agrícola conocen casos de afectaciones a la salud. Dejando de lado las intoxicaciones agudas, los «expertos» ${ }^{26}$ asocian a los agroquímicos casos de dermatitis, conjuntivitis, alergias, dolores de cabeza crónicos, e incluso, enfermedades relacionadas con la afectación del sistema inmunológico, embarazos sin embrión, cánceres diversos y depresión. En cuanto al resto de entrevistados, hay mucha confusión sobre las enfermedades asociadas a los agroquímicos debida principalmente a la falta de información. En este sentido, las enfermedades más reportadas, exceptuando también las intoxicaciones agudas, son el cáncer, los problemas de piel y vista, la esterilidad, las malformaciones, las alergias y la diabetes.

Yo solo sé el caso de una paciente, bueno, de dos, que su esposo era papayero y había tenido cinco abortos consecutivos, y finalmente se lo atribuyeron al problema del tóxico de la papaya. Tuvo que dejar de cultivar papaya [el marido], así fue como se pudo quedar embarazada y ahora tiene un bebé. El otro caso es el de una niña, hija de un papayero, igual, con leucemia linfoblástica [...], muy lento dicen, pero progresivo, porque lo absorbió la niña. Pues sí, como usted dice, hemos tenido un aumento de casos de cáncer, pero en sí son muy pocos los que se documentan (GJG, médico).

A ese amigo lo que le pasó es que la vista se le fue terminando y llegó un día en que ya miraba muy poco. Él se dedicaba a trabajar de jornalero en la papaya y no le dieron nunca protección ni nada (EJM, agricultor).

26 En este caso me refiero a las autoridades médicas entrevistadas. 
La multicausalidad reconocida de las enfermedades no puede obviar la presencia de tóxicos industriales y agrícolas dentro de nuestro cuerpo y en la biosfera, lo que lleva a los investigadores a acuñar nuevas expresiones como «cuerpos tóxicos» $u$ «obesos ambientales» (Porta, 2009; n. d.). El Dr. Porta, en un estudio realizado en Almería en 2008, concluye que la mayoría de los productos agrícolas analizados presentan contaminación por plaguicidas, y al ser sustancias que actúan como disruptores endocrinos, persistentes y bioacumulables, los límites legales establecidos por la normativa no garantizan la protección de la salud pública (Porta et alii, 2008). Así pues, no hay duda de que lo que los agricultores vierten al campo lo acaban encontrando los médicos en el cuerpo humano. Una de las conclusiones más relevantes del estudio es la sospecha de que los compuestos orgánicos persistentes (COP) pueden contribuir en muchas patologías, como la infertilidad, las alteraciones neurodegenerativas, el cáncer, y tener un papel (co)causal en el asma y la diabetes (ibid.: 25). En este sentido, la mayoría de los agricultores entrevistados consideran que el riesgo para la salud asociado tanto a la actividad productiva como a la ingesta de los productos del campo es muy similar, representando un $45 \%$ de las respuestas, y los que lo atribuyen solo a la actividad productiva representan un $25 \%$ de los entrevistados. Pero si diferenciamos los agricultores en orgánicos e industriales, los primeros consideran mayoritariamente (80\%) que hay riesgo tanto en la producción como en el consumo, y los segundos priorizan la producción en un 33,3\% de los casos. En cuanto al grupo de activistas, estos siguen la misma tendencia (el $62 \%$ de los entrevistados considera igual de riesgoso el consumo del alimento que la producción del mismo), y, en cambio, lo que difiere es el punto de vista de las autoridades, que perciben como principal vía de exposición y riesgo de los agroquímicos el ámbito productivo. Por lo tanto, los discursos de los agricultores y activistas son los que presentan una mayor asociación entre salud e inocuidad alimentaria.

El otro día compramos unos melones que sabían mucho a veneno [se ríe] y papaya también. Hay más riesgo cuando se come la fruta, aunque fumigar la fruta también es riesgoso (MHA, agricultor). 
Yo creo que hay más casos de enfermedades por consumo, pero es más alarmante los casos agudos, que son menos. La contaminación por consumo, hay muchos, pero no se sabe tanto porque es lento (AV, técnico de SEDAPA) ${ }^{27}$.

Otros estudios sobre los efectos de los plaguicidas en la salud y el medio ambiente dicen que la exposición a plaguicidas puede desembocar en la participación de sus ingredientes activos en diferentes enfermedades metabólicas, de desarrollo, reproductivas, de toxicidad pulmonar, neurotóxicas o en carcinogénesis (Calderón-Garcidueñas et alii, 2018; Castillo-Castañeda et alii, 2016; Suárez-Jacobo et alii, 2017; Giulivo et alii, 2016; IARC/WHO, 2017; Fénichel y Chevalier, 2017; Loomis et alii, 2015), o en enfermedades neurodegenerativas, autismo, asma, síndrome metabólico y problemas de fertilidad (Pelallo Martínez, 2010; Quintanilla, 2017; Xiao, Clark y Park, 2017). El alcance del riesgo de los agroquímicos no termina en el ámbito productivo, sino que también comprende el ámbito alimentario, ya sea por los alimentos directamente contaminados con residuos de plaguicidas o por la contaminación de la cadena trófica. En este sentido, la Associaçao Brasileira de Saúde Colectiva (ABRASCO, 2015) habla de inseguridad alimentaria en referencia a los alimentos contaminados por ingredientes activos de plaguicidas, y en el caso de México, uno de los estudios más recientes sobre el riesgo de los plaguicidas en las hortalizas es el de Pérez, Navarro y Miranda (2013), una revisión de las diferentes publicaciones sobre la presencia de plaguicidas en hortalizas que resalta la presencia de plaguicidas no autorizados o prohibidos en ciertos cultivos, así como la presencia de residuos de plaguicidas en más del 50\% de las muestras evaluadas de hortalizas y frutas. En un estudio realizado en Veracruz (México), se muestrearon 150 individuos para detectar los niveles de residuos organoclorados en el tejido adiposo y todos presentaron niveles de contaminación asociados al consumo de alimentos contaminados.

27 Secretaría de Desarrollo Agropecuario, Pesca y Acuacultura (<http://www.sedapa.oaxaca.gob. $\mathrm{mx} / \mathrm{)}>$. 
Los efectos de los plaguicidas no solo ponen en peligro la salud humana $^{28}$, sino que también afectan el medio ambiente ${ }^{29}$. Por ejemplo, hay estudios sobre las implicaciones de los COP presentes en los plaguicidas organoclorados sobre la mortalidad masiva y la presencia de cáncer en peces, como los trabajos de Vargas (2015), Gold-Bouchot y Zapata-Pérez (2010) y Martínez Villa et alii (2014), o estudios sobre las afectaciones al ambiente y los receptores ecológicos en general (González, 2010; Arellano-Aguilar et alii, 2017). En cualquier caso, para una revisión más exhaustiva del estado actual de la investigación sobre los efectos al medio ambiente y la salud humana de los plaguicidas en México se puede consultar el trabajo de García Hernández et alii (2018). En este se ponen de relieve los PAP utilizados en el país (paratión metílico, malatión, paraquat, glifosato $^{30}$, etc.), los efectos de estos plaguicidas en trabajadores agrícolas (alteraciones genéticas, disminución de la calidad del semen, alteración del perfil hormonal, mayor riesgo de anencefalia, etc.), así como los efectos en el ambiente (concentraciones de DDT en los huevos de tortuga carey de la costa de Campeche, etc.). El estudio concluye que existe suficiente evidencia para determinar que el uso de plaguicidas, tanto histórico como actual, está asociado al deterioro de los ecosistemas y de la salud de la población de México.

\subsection{Las alternativas al modelo agrícola industrial}

Los retos que plantea la adopción de técnicas y estrategias agrícolas alternativas en la zona de estudio están siendo encabezados principalmente por asociaciones civiles: desde el fomento de la agricultura orgánica al establecimiento de redes comerciales locales, pasando por el empoderamiento productivo (mediante formaciones, ayudas para cultivar horta-

28 Véase: <https://www.getholistichealth.com/78337/gluten-intolerant-glyphosate/?fbclid=IwARopgK g4L15xLmBS_DLpCmadmwN4eM-ozZgm_74436Q4Z5XpudSJcOMhZgQ> (última consulta: 28-3-2019). 29 Véase: <https://www.lajornadamaya.mx/2018-06-26/En-Yucatan-se-regala-cancer--con-apoyos-estatales-de-agroquimicos $>$; http://contrapoderweb.com/2018/o2/19/cofirmado-los-peces-mueren-por-envenenamiento-y-no-por-la-temperatura-videos/?fbclid=IwARofSFMEZBIZEG2lJN5GtWpxLoqE-X7TYSRhWstMo8zFDDzGsyVum1QsIgo> (última consulta: 27-3-2019).

30 Recientemente prohibido en Austria por su posible efecto cancerígeno. Véase: <https://www.reuters.com/article/us-austria-glyphosate/austria-set-to-be-first-eu-country-to-ban-all-uses-of-weedkiller-glyphosate-idUSKCNiTXIJR> (última consulta: 18-8-2019). 
lizas, pase en cadena ${ }^{31}$ ) y la conciencia ambiental (reservas celulares ${ }^{32}$, programa de reciclaje de envases, uso de controladores biológicos). De todos modos, aún falta un largo camino por recorrer, porque la representatividad de estas iniciativas en el territorio es todavía escasa. En la costa de Tututepec, ECOSTA ${ }^{33}$ y la Junta Local de Sanidad Vegetal son las entidades locales que trabajan para avanzar hacia modelos agrícolas más sostenibles. A nivel estatal es importante el trabajo de RAPAM ${ }^{34}$, que lucha para lograr cambios en las políticas públicas a favor del control agroecológico de plagas, para la protección de los derechos a una alimentación sana, libre de plaguicidas y transgénicos, por la soberanía alimentaria y por un medio ambiente libre de contaminantes (Bejarano et alii, 2017).

Así, la principal alternativa al modelo agrícola industrial se fundamenta en la promoción de la agricultura orgánica (o natural), en tanto que representa la cara opuesta a la agricultura industrial en lo que respeta al uso de agroquímicos sintéticos, sin excluir otros modelos agrícolas sostenibles de la región, como la agricultura campesina ${ }^{35}$. Aunque la mayoría de los agricultores entrevistados relacionan la seguridad alimentaria con «el alimento no contaminado» o «sin químicos», la agricultura orgánica no se valora como una opción viable porque prevalece la mirada comercial.

Sabemos que podemos usar el orgánico, pero es más lento y más caro, se nos encarece nuestro producto y al final no nos lo retribuyen, no tenemos mercado cerca o no tenemos cómo comercializar ese producto orgánico (TL, agricultor).

\footnotetext{
31 Proyecto llevado a cabo por Ecosta en los últimos cinco años que consistía en entregar de forma gratuita dos animales (a escoger entre cerdos, gallinas o cabras) a la familia que se comprometiera a cultivar hortalizas para su autoconsumo. Además, la familia debía comprometerse a regalar una cría de los animales recibidos a otra familia, tan pronto fuera posible.

32 Proyecto de concienciación sobre la importancia de cuidar el bosque para que se convierta en una reserva de recursos para nosotros, como agua, plantas medicinales, leña, etc. Fue el proyecto pionero de Ecosta.

33 La actividad más transversal de la asociación sería la de asesorar y formar en el ámbito de la agricultura orgánica, promoviendo canales de comercialización de productos agrícolas sin químicos y de productores locales, como los proyectos de algodón y maíz criollo orgánicos.

34 Acrónimo de Red de Acción sobre Plaguicidas y Alternativas en México.

35 Las características principales de este tipo de agricultura son la diversidad biológica, el aprovechamiento de los recursos naturales de la zona, el policultivo y la utilización de semilla propia, heredada o intercambiada.
} 
Solo 5 del total de agricultores entrevistados (20) practican la agricultura orgánica. En general, los riesgos para la salud permanecen invisibles para los mismos agricultores, pero también para el conjunto de la sociedad. El trabajo etnográfico de Saxton (2015) evidencia la invisibilización de los riesgos asociados a los agroquímicos debido a la posición social marginada de los agricultores, y partiendo de las teorías de la ecología política, considera la fuerza organizativa de los movimientos sociales como la única opción viable para liderar la creación de nuevas alternativas al modelo agrícola. Pero estas nuevas alternativas deberían incluir una nueva relación hombre-naturaleza, ya que los riesgos ambientales solo se asocian a la afectación del rendimiento productivo, y para lograr percibir el riesgo ambiental en asociación con el riesgo a la salud sin duda sería necesario concebir la interrelación con el medio ambiente de una forma más integral. Entender la agricultura más allá del producto que se cultiva implicaría tener en cuenta el conjunto del ecosistema y nuestro lugar dentro de este.

Uno de los principales hándicaps que condiciona el modelo agroalimentario mexicano es el mercado nacional y su funcionamiento a nivel estructural. Toda la producción agrícola de Oaxaca, Chiapas, Tabasco y Veracruz se centraliza en la central de abastos de Ciudad de México y desde allí retorna a los diferentes estados. La central de abastos funciona como un monopsonio, con muy pocos compradores y sus respectivos acopiadores, que fijan el precio al que comprarán el producto. Todo el limón y la papaya producidos en la zona de estudio se comercializan de esta manera, sin posibilidad de que exista un mercado alternativo local y de proximidad que garantice precios más justos.

En este mercado no está permitido entrar como un campesino suelto, sino que trae el producto el acopiador. Este te va a decir a qué precio te lo va a comprar, si no te lo deja y a ver tú dónde lo vendes. Ese comprador te lo va a comprar mal, pero es el único que te lo va a comprar. Es una locura, pero está organizado a través del poder de estos acopiadores (ADI, activista).

Estas relaciones macroestructurales que rigen el funcionamiento del mercado nacional condicionan la situación socioeconómica de la pobla- 
ción de la zona de estudio. Las necesidades de la población (también las de salud) están mediatizadas por las posibilidades y los límites establecidos por los niveles de productividad económica, social e ideológica, pero también por el «sistema de transacciones» entre los sectores hegemónicos y los subalternos, que se desarrollan mediante canales institucionales legitimados y establecidos por las clases dominantes (Menéndez, 1981). De este modo, las relaciones entre las comunidades agrícolas, las empresas agroexportadoras, las industrias de agroquímicos y las instituciones gubernamentales se tejen mediante actos de dominación simbóli$\mathrm{Ca}^{36}$, donde los agroquímicos se convierten en el elemento central para transfigurar las relaciones de dominación y explotación en relaciones de cooperación, asistencia y desarrollo.

Por el momento, el camino está trazado y la impronta de las asociaciones locales avanza paso a paso con los proyectos de concienciación ambiental, de formación en agricultura orgánica y de promoción de métodos de control de plagas más sostenibles, como los controladores biológicos.

\section{Conclusiones}

El trabajo de investigación parte de la premisa de que las ideas y experiencias de las personas sobre una técnica o aplicación construyen sus representaciones sociales del riesgo, entendidas como aquellos conceptos relativos a objetos cuyo sentido es construido y compartido de forma colectiva (Gracia, 2004: 131). La percepción del riesgo asociado a los agroquímicos está condicionada por las creencias socioculturales propias de la costa de Oaxaca y por las experiencias de intoxicación vividas o compartidas, más que por el «conocimiento científico» sobre las repercusiones en la salud humana y el medio ambiente que se derivan del uso de los productos agroquímicos.

Las representaciones en el ámbito agrícola de las comunidades costeras de Tututepec refuerzan y justifican la adopción de prácticas agrarias «arriesgadas» relacionadas con el uso intensivo de agroquímicos y la falta de protección individual. Las categorías flojo y débil tiñen las relaciones entre los diferentes agentes sociales, principalmente entre los mismos

36 Tomo como referencia la teoría del intercambio de obsequios de Bourdieu (2007). 
agricultores, y nos conectan con la construcción de una identidad masculina que afronta el peligro basándose en una concepción de inmunidad subjetiva (Douglas, 1996). Esta exposición al riesgo se ve agravada en contextos de marginalidad elevada, donde la mayoría de los agricultores que se dedican a las tareas de fumigación provienen de las comunidades de la sierra (zonas mayoritariamente indígenas), y donde el estigma de la «debilidad masculina» frente la manipulación y aplicación de agroquímicos se suma al racismo todavía presente hacia los indígenas. Así pues, la situación de vulnerabilidad social en el ámbito agrícola no solo viene determinada por el contexto socioeconómico y los factores socioculturales, sino que es reforzada por el estigma étnico (Gall, 2004).Gall, 2004).

El tiempo de manifestación de los malestares (normalmente corto), la regularidad en que se presentan, las características sintomáticas y el contexto sociocultural (etnia, edad, género, etc.) influyen en las trayectorias terapéuticas escogidas. Solo los casos graves, con una afectación física importante, utilizarán los servicios biomédicos disponibles en la región, y la gran mayoría utilizarán los «tratamientos profanos» (zumo de limón, leche, chaya), se automedicarán o se autorregularán los niveles de «químicos» absorbidos en el cuerpo. Este hecho incrementa la invisibilización de los riesgos de los agroquímicos en la salud y no permite contabilizar oficialmente el número de casos reales de intoxicación.

En general, el incremento de las enfermedades metabólicas en la zona de estudio no se vincula con la elevada utilización y presencia de agroquímicos, a pesar de las cada vez más numerosas evidencias científicas que les otorgan un papel relevante en enfermedades como la diabetes, la obesidad y el cáncer (Rylander, Rignell-Hydbom y Hagmar, 2005; Donato y Zani, 2010; Uemura, 2012; Ruzzin, 2012). Este hecho podría deberse a una cierta ignorancia sobre las enfermedades ocupacionales por parte de las instituciones y de la «naturalización» de los problemas de salud derivados del trabajo agrícola por parte de los agricultores (Menéndez, 1981). En cualquier caso, es evidente que se deberían tomar medidas para eliminar los impactos de los agroquímicos como asunto de salud pública ${ }^{37}$, ya

37 Véanse: <https://www.endocrine.org/search/\#q=agrochemicals>; <https://academic.oup.com/ edrv/issue/36/6>; <https:/www.bioecoactual.com/2018/06/15/aumenta-numero-pesticidas-disrup- 
sea mediante el desarrollo de pesticidas orgánicos, la gestión integral de plagas o la transformación del sistema agroalimentario actual.

La percepción del riesgo de los agroquímicos sobre la salud toma distintas formas, desde la intoxicación aguda a la incertidumbre sobre los efectos toxicológicos de estos, pasando por la falta de hábitos de protección que nos exponen a sus peligros. Pero estos discursos no se articulan suficientemente con la adopción de nuevas prácticas a favor de una agricultura más sostenible, a pesar de las iniciativas promovidas por las asociaciones civiles. A excepción de los agricultores orgánicos entrevistados, quienes asocian el riesgo sobre la salud con la calidad alimentaria y la contaminación del medio ambiente, el resto de agricultores no conciben una agricultura sin un elevado rendimiento productivo para cubrir las necesidades del mercado mexicano, hecho que condiciona la actividad productiva y la ata al uso intensivo de agroquímicos. En este sentido, serían necesarios cambios estructurales dentro del sistema de transacciones establecido por los diferentes agentes que intervienen en el sistema agroalimentario, así como fomentar la presencia de las iniciativas a favor del fomento de la agricultura orgánica en la zona de estudio.

Ante la situación de crisis ecológica que sufre el planeta, la crítica surgida desde la ecología política ha puesto en evidencia la ineficiencia de los sistemas productivos «modernos», que generan elevados costes ecológicos (y de salud), haciendo emerger una avalancha de investigaciones y publicaciones (Altieri, 2014; Altieri y Toledo, 2010; Giraldo y Rosset, 2016; Patel, 2009; Rosset, 2003; Rosset et alii, 2016) que coinciden en que los conocimientos, tecnologías y estrategias productivas de los campesinos son los ecológicamente adecuados y los que se han de tomar como punto de partida en la construcción de las alternativas agroecológicas reivindicadas por los movimientos sociales de justicia global y entidades como la Vía Campesina, ETC Group, Grain y PAN.

El reto de afrontar los agroquímicos representa afrontar las estructuras y lógicas que gobiernan la sociedad contemporánea y las operaciones empresariales orientadas al beneficio (Saxton, 2015). El aumento de

tores-endocrinos-alimentos/?fbclid=IwARorNIUQbxbNYW5aefxbZoRglDFDt4T9hnbMr2dInxoxhb-ydPYqlTnKmgc> (última consulta: 27-3-2019). 
enfermedades crónicas en la sociedad actual nos reta a tomar medidas «radicales» en el ámbito de la contaminación ambiental, que superen la teoría de los «límites máximos permitidos» y el reduccionismo del análisis coste-beneficio, y nos emplace en el escenario de la reivindicación de los derechos humanos. El presente trabajo pretende hacer visible una de las muchas capas de sufrimiento ambiental que padecen los agricultores, poniendo en valor las representaciones sociales del riesgo alrededor del uso y presencia de los agroquímicos en el ámbito laboral y alimentario. Las aportaciones del estudio evidencian la necesidad de un cambio en las políticas socioambientales y agroalimentarias, poniendo de manifiesto un problema de salud pública relacionado con el mal uso y manipulación de agroquímicos y vinculado con la elevada incidencia del síndrome metabólico de la región.

\section{Bibliografía}

Alfie Cohen, M. (2017). «Riesgo ambiental: la aportación de Ulrich Beck». Acta Sociológica, 73, 171-194.

Altieri, M. A. (2014). «Agroecología, pequeñas fincas y soberanía alimentaria». Ecología Política, (38), 25-35.

Altieri, M. A. , y Toledo, V. (2010). «La revolución agroecológica de América Latina: Rescatar la naturaleza, asegurar la soberanía alimentaria y empoderar al campesino». El Otro Derecho, (42), 163-202.

Arellano, E.; Camarena, L.; Glascoe, C. von y Daesslé, W. (2009). «Percepción del riesgo en salud por exposición a mezclas de contaminantes». Revista Facultad Nacional de Salud Pública, Universidad de Antioquia Colombia, 27 (3), 291-301.

Arellano-Agullar, O.; Betancourt-Lozano, M.; Aguilar-Zárate, G. y Ponce DE LEON-Hill, C. (2017). «Agrochemical loading in drains and rivers and its connection with pollution in coastal lagoons of the Mexican Pacific». Environmental Monitoring and Assessment, 189 (6), 270.

Auyero, J. y Swistun, D. (2007). «Expuestos y confundidos. Un relato etnográfico sobre sufrimiento ambiental». Iconos. Revista de Ciencias Sociales, 28, 137-152.

Bauman, Z. (2001). La postmodernidad y sus descontentos. Madrid: Akal. 
BECK, U. (1998). La Sociedad del Riesgo. Hacia una nueva modernidad (3. . ed.). Barcelona: Paidós.

BECK, U. (2002). La sociedad del riesgo global. Madrid: Siglo XXI de España Editores, SA.

Begueria, A. (2016). Un equilibrio imperfecto. Alimentación ecológica, cuerpo y toxicidad. Barcelona: Editorial UOC.

Bejarano, F.; Colín, C.; GonzÁlez, I. G. ... y Kubiak, W. (2017). Los Plaguicidas Altamente Peligrosos en México. México: RAPAM.

Busqueta, J.; Etxezarreta, M.; Iglesias, J.; Navarro, F.; Presas, O.; Puig, A. y TeIxidó, J. (2017). «Apuntes sobre Marx y Naturaleza». Taifa, informes de economía crítica, 12.

Calderón-Garcidueñas, A. L.; Waliszewsisi, S. M.; Ruiz-Ramos, R., y del Carmen Martínez-Valenzuela, M. (2018). «Time trend tendency (19882014 years) of organochlorine pesticide levels in the adipose tissue of Veracruz inhabitants». Environmental Monitoring and Assessment, 190 (4), 206.

Calvario Parra, J. E. (2007). «Masculinidad, riesgos y padecimientos laborales. Jornaleros agrícolas del poblado Miguel Alemán, Sonora». Región y Sociedad, 19 (40), 39-72.

CARNeIro, F. F. (2015). Dossiê ABRASCO: um alerta sobre os impactos dos agrotóxicos na saúde. Associação Brasileira de Saúde Coletiva - ABRASCO. Sao Paulo: Expressao Popular.

Castillo-Castañeda, P. C.; Gaxiola-Robles, R.; Méndez-Rodríguez, L. C.; Labrada-Martagón, V. y Zenteno-Savín, T. (2016). «Antioxidantes, especies reactivas de oxígeno y daño oxidativo asociado a la presencia de plaguicidas organoclorados en la leche materna». Nutricion hospitalaria, 33 (2), 127.

CeCCAM у BRöт (2015). Conservación oficial y extractivismo en México. Ciudad de México.

CECCAM y BRÖT (2017). Treinta años de transgénicos en México (compendio cartográfico). Ciudad de México.

Ceciproc y Ecosta (2018). Propuesta de política pública en relación a la agenda 2030 de desarrollo sostenible (borrador). Villa de Tututepec de Melchor Ocampo. 
CONEVAL (2015). Medición de la pobreza a escala municipal 2010-2015. Ciudad de México. Disponible en: <https://www.coneval.org.mx/Medicion/Paginas/consulta_pobreza_municipal.aspx>.

Departament de Medi Ambient (2001). «Influència del medi ambient en la salut humana». Mediambient, Tecnologia i Cultura, 31.

Díaz-Barriga, F. (1996). «Los residuos peligrosos en México». Salud Pública Mex, 38 (2405), 280-291.

Dirección General de Epidemiología (2013). «Panorama histórico de morbilidad y mortalidad por Intoxicación por plaguicidas en México 19952012». Boletín Epidemiológico, 30 (34).

Donato, F. y ZANI, C. (2010). «Chronic exposure to organochlorine compounds and health effects in adults: diabetes and thyroid diseases». Annali di igiene: medicina preventiva e di comunita, 22 (3), 185-98.

Douglas, M. (1973). Pureza y peligro. Un análisis de los conceptos de contaminación y tabú. Madrid: Siglo XXI.

Douglas, M. (1996). La aceptabilidad del riesgo según las ciencias sociales. Barcelona: Paidós.

Douglas, M. y Wildavsky, A. (1983). Risk and culture. An essay on the Selection of Technological and Environmental Dangers. Berkeley; Los Angeles: University of California Press.

Elvia BARAJAS, R. (1991). «Biotecnología y revolución verde: Especificidades y divergencias». Sociológica. Revista del Departamento de Sociología de la Universidad Autónoma Metropolitana, 6 (16).

FAO, FIDA, OMS, PMA y UNICEF (2017). El Estado de la Seguridad Alimentaria y la Nutrición en el Mundo. Fomentando la resiliencia en aras de la paz y la seguridad alimentaria. Roma. Disponible en: <http://www.fao.org/ state-of-food-security-nutrition/es/>.

FÉNICHel, P. y CheVAlier, N. (2017). «Environmental endocrine disruptors: New diabetogens?» Comptes Rendus Biologies, 340 (9-10), 446-452.

Flocks, J.y Monaghan, P. (2003). «Collaborative Research with Farmworkers in Environmental Justice». Practicing Anthropology, 25 (1), 6-9.

Freitas, C.; Minayo, C.; Pena, P. y Santos, N. (2009). «Un ambiente enfermo: significados de la contaminación industrial en Isla de Maré, Bahía, Brasil». Desacatos, (39), 73-88. 
GALL, O. (2004). «Identidad, exclusión y racismo: reflexiones teóricas y sobre México». Revista mexicana de sociología, 66 (2), 221-259.

García Hernández, J.; Leyva Morales, J. B.; Martínez Rodríguez, I. E. ... y Perera Ríos, J. H. (2018). «Estado actual de la investigación sobre plaguicidas en México». Revista Internacional de Contaminación Ambiental, 34 (espo1), 29-60.

Giddens, A. (1994). Les consequences de la modernité. Paris: L'Harmattan.

Giraldo, O. F. y Rosset, P. M. (2016). «La agroecología en una encrucijada: entre la institucionalidad y los movimientos sociales». Guaju, 2 (1), 14. Giulivo, M.; López de Alda, M.; Capri, E. y Barceló, D. (2016). «Human exposure to endocrine disrupting compounds: Their role in reproductive systems, metabolic syndrome and breast cancer. A review». Environmental Research, 151, 251-264.

Gold-Bouchot, G. y ZaPata-PÉrez, O. (2010). Diagnóstico de la situación ambiental de los COP en el Golfo de México. Quintana Roo. Disponible en: https://doi.org/10.13140/2.1.4491.5202.

GonZÁlez, D. J. (2010). Aplicación de una metodología de evaluación de riesgo ecológico en fauna acuática de Coatzacoalcos, Veracruz. Tesis doctoral, Facultad de Ciencias Químicas, Ingeniería y Medicina, Universidad Autónoma San Luis de Potosí. Disponible en: <http://ninive.uaslp.mx/ jspui/bitstream/i/3584/1/DJGM-Tesis Doctorado.pdf $>$.

Gracia Arnaiz, M. (coord.) (2002). Somos lo que comemos. Estudios de alimentación y cultura en España. Barcelona: Editorial Ariel, SA.

Gracia Arnaiz, M. (2004). «Pensando sobre el riesgo alimentario y su aceptabilidad: el caso de los alimentos transgénicos». Revista de Nutrição, 17 (2), 125-149.

GRAIN (2011). «Alimentos y cambio climático: el eslabón olvidado». Disponible en: <https:/www.grain.org/es/article/entries/4364-alimentos-y-cambio-climatico-el-eslabon-olvidado>.

IAASTD (2009). «Global Report. Agriculture at a Crossroads». Disponible en: <https://www.globalagriculture.org/fileadmin/files/ weltagrarbericht/IAASTDBerichte/GlobalReport.pdf>.

IARC/WHO (2017). "DDT, Lindane, and 2,4-D». IARC monographs on the evaluation of carcinogenic risks to humans; volume 113. 
INEGI (2015). «México en cifras». Disponible en: <http://www.beta.inegi. org.mx/app/areasgeograficas/?ag=20\#>.

KALLIS, G. (2012). «Ecomodernismo versus ecología política». Ecologiapolitica.Info, (2), 22-24. Disponible en: <http:/www.ecologiapolitica.info/ wp-content/uploads/2015/12/050_Kallis_2016.pdf>.

Karam, M. Á.; Ramírez, G.; Montes, L. P. B.; Galván, J. M. ... y Manuel, J. (2004). «Plaguicidas y salud de la población». Red de revistas Científicas de América Latina, el Caribe, España y Portugal, 11, 246-254.

KÜSTER, Á. S. (2016). Volver a la tierra. Transiciones agroecológicas de los sistemas agroalimentarios hacia la soberanía alimentaria. Tesis doctoral, Instituto Interuniversitario de desarrollo local, Universidad de Valencia. Disponible en: <https://dialnet.unirioja.es/servlet/tesis?codigo=79238>.

Lara Millán, G. y Reyes Cruz, H. (2010). Maíces criollos de Tututepec: cultura, genes y políticas públicas. México: Ecosta Yutu Cuii.

Little, P. C. (2012). «Another Angle on Pollution Experience: Toward an Anthropology of the Emotional Ecology of Risk Mitigation». Ethos, 40 (4), 431-452.

Loomis, D.; Guyton, K.; Grosse, Y. ... y Straif, K. (2015). «Carcinogenicity of lindane, DDT, and 2,4-dichlorophenoxyacetic acid». The Lancet. Oncology, 16 (8), 891-892.

Martínez-Hernáez, Á. (2011). Antropología Médica. Teorías sobre la cultura, el poder y la enfermedad. Barcelona: Anthropos editorial.

Martínez Alier, J. (2006). El ecologismo de los pobres (2. a ed.). Barcelona: Icaria editorial, $\mathrm{SA}$.

Martínez Villa, G.; Inzunza, J. R.; Tolentino, V. A. ; ... Y GonzÁlez Medina, G. (2014). «Contenido de plaguicidas organoclorados en varios peces depredadores de la costa de Oaxaca y evaluación del riesgo de exposición por consumo en la salud humana». En A. V. Botello, F. L. Páez-Osuna, S. Betancourt-Lozano ... y R. Lara-Lara (ed.). Pacífico Mexicano. Contaminación e Impacto Ambiental: Diagnóstico y Tendencias (pp. 169-208). Mazatlán: UAC, UNAM-ICMYL, CIAD-MAZATLÁN, CIBNOR, CICESE. Menéndez, E. (1981). Poder, estratificación y salud. Análisis de las condiciones sociales y económicas de la enfermedad en Yucatán. México: Ediciones de la Casa Chata. 
Menéndez, E. (1998). «Estilos de vida, riesgos y construcción social. Conceptos similares y significados diferentes». Estudios sociológicos, 16 (46), 37-67.

MenÉNDEZ, E. (2009). De sujetos, saberes y estructuras: introducción al enfoque relacional en el estudio de la salud colectiva. Buenos Aires: Lugar Editorial. Montagut, X. y Vivas, E. (2009). Del campo al plato. Barcelona: Icaria editorial.

ONU A/HRC/34/48 (2017). Informe de la Relatora especial sobre el derecho a la alimentación. Disponible en: <http://acnudh.org/wp-content/uploads/2018/03/G1701976.pdf>.

Ortega-Ceseña, J., Espinosa-Torres, F. y López-Carrillo, L. (1994). «El control de los riesgos para la salud generados por los plaguicidas organofosforados en México: retos ante el Tratado de Libre Comercio». Salud Pública de México. Disponible en: <https://doi.org/10.1017/ CBO9781107415324.004>.

Parlamento Europeo y Consejo de la Unión Europea (2008). Reglamento (CE) $n .{ }^{\circ} 1272 / 2008$ de 16 de diciembre de 2008 sobre clasificación, etiquetado y envasado de sustancias y mezclas, y por el que se modifican y derogan las Directivas 67/548/CEE y 1999/45/CE y se modifica el Reglamento (CE) n. ${ }^{\circ}$ 1907/2006. Disponible en: <https://eur-lex.europa. eu/LexUriServ/LexUriServ.do?uri=OJ:L:2008:353:0001:1355:es:PDF>.

Patel, R. (2009). «What does food sovereignty look like?» Journal of Peasant Studies, 36.

Pelallo Martínez, N. A. (2010). Evaluación de riesgo en salud en población infantil expuesta a una mezcla de contaminantes en Coatzacoalcos, Veracruz. Tesis doctoral, Universidad Autónoma de San Luis Potosí.

Pérez, M. A.; Navarro, H. y Miranda, E. (2013). Residuos de plaguicidas en hortalizas: Problemática y riesgo en México. Revista Internacional de Contaminación Ambiental, 29, 45-64.

Porta, M. (n. d.). «Contaminants en el cos humà. Els tòxics industrials i agrícoles al nostre cos per terra, mar i aire»S. Disponible en: <http:// forumdedebats.vicentitats.cat/diba_projecte/miquel-porta-contaminants-en-el-cos-huma-els-toxics-industrials-i-agricoles-al-nostrecos-per-terra-mar-i-aire/>. 
Porta, M.; Kogevinas, M.; Zumeta, E.; Sunyer, J.; Ribas-Fitó, N. y colaboradores (2002). «Concentraciones de compuestos tóxicos persistentes en la población española: el rompecabezas sin piezas y la protección de la salud pública». Gaceta Sanitaria, 16 (3), 257-266.

Porta, M.; Gasull, M.; Puigdomènech, E. y Bosch de Basea, M. (2008). La influencia de la dieta en las concentraciones corporales de Compuestos Orgánicos Persistentes. Barcelona: IMIM y UAB.

Porta, M. (2009). «Cuerpos tóxicos». Culturas La Vanguardia, 388, 2-5.

Quintanilla, B. (2017). «Exposición a plaguicidas y salud humana». En Simposio Nacional de Plaguicidas desde una Perspectiva Bioética. Querétaro: CINVESTAV, Departamento de Toxicología. Documento electrónico. R-PLAFEST (2014). Reglamento en Materia de Registros, Autorizaciones de Importación y Exportación y Certificados de Exportación de Plaguicidas, Nutrientes Vegetales y Sustancias y Materiales Tóxicos o Peligrosos. México: Diario Oficial de la Federación.

Reith, G. (2004). «Uncertain Times». Time E Society, 13 (2-3), 383-402.

Rosset, P. (2003). «Soberanía Alimentaria : Reclamo Mundial del Movimiento Campesino». Food Sovereignty: Global Rallying Cry of Farmer Movements, 9 (4), 1-5.

Rosset, P., LVC y Drago, M. (2016). «La agroecología como resistencia y Transformación». Boletín nyeleni, 28, 1-6.

Ruzzin, J. (2012). «Public health concern behind the exposure to persistent organic pollutants and the risk of metabolic diseases». BMC Public Health, 12 (1), 298.

Rylander, L.; Rignell-Hydbom, A. y Hagmar, L. (2005). «A cross-sectional study of the association between persistent organochlorine pollutants and diabetes». Environmental Health, 4 (1), 28.

SAXTON, D. I. (2015). «Ethnographic movement methods: anthropology takes on the pesticide industry». Journal of Political Ecology, 22, 368-387. Toledo, V. M. (2013). «El metabolismo social: una nueva teoría socioecológica». Relaciones, 136, 41-71.

Toledo, V. M.; Ortiz-Espejel, B. F.; Cortés, L.; Moguel, P. y Ordoñez, M. de J. (2003). «The Multiple Use of Tropical Forests by Indigenous Peoples 
in México: a Case of Adaptive Management». Conservation Ecology, 7 (3), art9.

Uemura, H.(2012). «Associations of exposure to dioxins and polychlorinated biphenyls with diabetes: based on epidemiological findings». Nihon eiseigaku zasshi. Japanese journal of hygiene, 67 (3), 363-374.

URquíA-FERnÁndeZ, N. (2014). «La seguridad alimentaria en México». Salud Pública de México, 56, 92-98.

VARgas Hernández, A. A. (2015). Impacto de compuestos orgánicos persistentes (COP's) en la zona sur del acuífero de Quintana Roo, México. Centro de Investigación Científica de Yucatán, AC. Tesis doctoral. Disponible en: $<$ https:/cicy.repositorioinstitucional.mx/jspui/bitstream/1003/365/1/ PCA_M_Tesis_2015_Vargas_Abraham.pdf>.

VIVAS, E. (2014). El negocio de la comida. Barcelona: Icaria editorial.

Widger, T. (2014). "Pesticides and global health: 'ambivalent objects' in anthropological perspective». Somatosphere. Disponible en: $<$ http://somatosphere.net/2014/08/pesticides-and-global-healthambivalent-objects-in-anthropological-perspective.html>.

$\mathrm{X}_{\text {IAO }}$ X.; CLARK, J. M. y PARK, Y. (2017). «Potential contribution of insecticide exposure and development of obesity and type 2 diabetes». Food and Chemical Toxicology, 105, 456-474. 
